Technische

Universität

Berlin

\author{
C. Vinod Chandran, Kai Volgmann, Suliman Nakhal, Reinhard \\ Uecker, Elena Witt, Martin Lerch, Paul Heitjans
}

\title{
Local Ion Dynamics in Polycrystalline beta-LiGaO2: A Solid-State NMR Study
}

Open Access via institutional repository of Technische Universität Berlin

\section{Document type}

Journal article | Published version

(i. e. publisher-created published version, that has been (peer-) reviewed and copyedited; also known as:

Version of Record (VOR), Final Published Version)

This version is available at

https://doi.org/10.14279/depositonce-12530

\section{Citation details}

Chandran, C. V., Volgmann, K., Nakhal, S., Uecker, R., Witt, E., Lerch, M., \& Heitjans, P. (2017). Local lon Dynamics in Polycrystalline -LiGaO2: A Solid-State NMR Study. In Zeitschrift für Physikalische Chemie (Vol. 231, Issues 7-8, pp. 1443-1453). Walter de Gruyter GmbH. https://doi.org/10.1515/zpch-2016-0920

\section{Terms of use}

This work is protected by copyright and/or related rights. You are free to use this work in any way permitted by the copyright and related rights legislation that applies to your usage. For other uses, you must obtain permission from the rights-holder(s). 


\title{
C. Vinod Chandran*, Kai Volgmann, Suliman Nakhal, Reinhard Uecker, Elena Witt, Martin Lerch and Paul Heitjans* Local Ion Dynamics in Polycrystalline $\beta$-LiGaO ${ }_{2}$ : Solid-State NMR Study
}

DOI 10.1515/zpch-2016-0920

Received October 21, 2016; accepted March 17, 2017

\begin{abstract}
Solid-state nuclear magnetic resonance spectroscopy is an efficient technique to characterize dynamics and structure of materials. It has been widely used to elucidate ion dynamics in lithium ion conductors. Fast moving lithium ions are needed in energy storage devices, whereas slow ion motion is exploited in some materials used, for example, as blankets in fusion reactors. $\beta$-lithium gallium oxide $\left(\mathrm{LiGaO}_{2}\right)$ is a slow $\mathrm{Li}^{+}$ionic conductor similar to $\gamma$-lithium aluminum oxide $\left(\mathrm{LiAlO}_{2}\right)$. In an ion conductor, in addition to the main diffusion process, localized motions (to-and-fro jumps) may be present. In the present work, with the help of solid-state NMR experiments, we report on the localized movements of $\mathrm{Li}^{+}$ionic species in $\beta$ - $\mathrm{LiGaO}_{2}$ in the temperature range between $300 \mathrm{~K}$ and $450 \mathrm{~K}$. In this work, we have mainly extracted the peculiarities of ion dynamics from ${ }^{7} \mathrm{Li}$ spin-alignment echo NMR measurements and the observation of the motional narrowing of the central transition signal of ${ }^{7} \mathrm{Li}$.
\end{abstract}

Keywords: $\beta$ - $\mathrm{LiGaO}_{2}$; ${ }^{71} \mathrm{Ga}$ NMR; ' $\mathrm{Li} \mathrm{NMR}$; Li ion diffusion; motional narrowing; solid-state NMR; spin-alignment echo.

\section{Introduction}

Lithium containing solid materials have several applications mainly based on the mobility of the Li ions within these systems. Lithiated solids with highly mobile

\footnotetext{
*Corresponding authors: C. Vinod Chandran and Paul Heitjans, Institut für Physikalische Chemie und Elektrochemie, Leibniz Universität Hannover, Callinstr. 3-3a, 30167 Hannover, Germany; and Zentrum für Festkörperchemie und Neue Materialien (ZFM), Leibniz Universität Hannover, Callinstr. 3-3a, 30167 Hannover, Germany, e-mail: vinod.nair@pci.uni-hannover.de (C.V.Chandran), heitjans@pci.uni-hannover.de (P. Heitjans)

Kai Volgmann and Elena Witt: Institut für Physikalische Chemie und Elektrochemie, Leibniz Universität Hannover, Callinstr. 3-3a, 30167 Hannover, Germany; and Zentrum für Festkörperchemie und Neue Materialien (ZFM), Leibniz Universität Hannover, Callinstr. 3-3a, 30167 Hannover, Germany
}

Suliman Nakhal and Martin Lerch: Technische Universität Berlin, Strasse des 17. Juni 135, 10623 Berlin, Germany

Reinhard Uecker: Leibniz-Institut für Kristallzüchtung, Max-Born-Strasse 2, 12489 Berlin, Germany 
Li ions have been used as efficient lithium ion battery (LIB) materials for decades [1-3]. But the materials with slow Li ion diffusion (e.g. lithium aluminate) are also employed, for example as tritium-breeder blankets for fusion reactors $[4,5]$, or as substrates for semiconductor growth [6]. The fundamental dynamic processes in slow ion conductors have much less been studied and are not yet fully understood. In such materials, in addition to the main slow ionic diffusion mode, there may be faster localized motions present. These localized (or to-and-fro) jumps of ions are usually difficult to observe with regular mobility or conductivity investigations. In the present study, we employed nuclear magnetic resonance (NMR) spectroscopy to monitor local ionic jumps, in which only a small part of the $\mathrm{Li}$ ions are involved.

There are several reports on solid-state NMR studies of materials with fast diffusing Li ions [7-11]. Most of them focus on materials used in LIB's and investigate their dynamics, structure and conductivity behaviors. But there are only a few reports available on NMR studies of materials with purely slow Li dynamic modes alone [12-14]. Witt et al. [13] studied the slow ionic diffusion in microcrystalline $(m-) \gamma$-lithium aluminate $\left(\mathrm{LiAlO}_{2}\right)$. From variable-temperature spinlattice relaxation (SLR) studies [15], spin-alignment echo (SAE) experiments [16] and conductivity measurements [17], a slow overall ionic diffusion with a diffusion coefficient of the order of $10^{-19} \mathrm{~m}^{2} \mathrm{~s}^{-1}$ at $473 \mathrm{~K}$ and an activation energy of $0.7 \mathrm{eV}$ were found. In addition, the $\mathrm{Li}^{+}$motion led to the averaging of dipolar broadening of the central transition ${ }^{7} \mathrm{Li}$ signal of $m-\mathrm{LiAlO}_{2}$. This motional narrowing ocurred in the temperature range between 500 and $900 \mathrm{~K}$. The fullwidth at half-maximum (FWHM) decreased from $7 \mathrm{kHz}$ to a few hundreds of $\mathrm{Hz}$ in this temperature range [13]. A reduction of FWHM of this order is usual for a complete motional narrowing process (involving all Li ions) leaving behind only the inhomogeneous line broadening. Recently, a substantial increase of ionic mobility has been observed on reduction of particle size in $\mathrm{LiAlO}_{2}$ by mechanical milling [18].

$\mathrm{LiGaO}_{2}$ is an interesting material, in which structural differences contribute significantly towards ionic conductivity. The high-pressure phase $\alpha$ - $\mathrm{LiGaO}_{2}$ [19] forms layered solid solutions with $\mathrm{LiCoO}_{2}$ [20], $\alpha$ - $\mathrm{LiAlO}_{2}$ [21], and $\mathrm{LiNi}_{1-x} \mathrm{Co}_{x} \mathrm{O}_{2}$ [22], which can be employed as potential electrode materials for Li-ion batteries. However, an ultra-slow Li ionic diffusion is expected for polycrystalline $\beta$ $\mathrm{LiGaO}_{2}$ similar to $\gamma$ - $\mathrm{LiAlO}_{2}$, despite their structural differences. But to probe such slow dynamics, the NMR experiments have to be carried out at very high-temperatures. However, localized motions can be probed at lower temperatures. In the present work, we report localized Li ion motion based on our observations of motional narrowing (between 293 and $473 \mathrm{~K}$ ) and SAE experiments (between 373 and $448 \mathrm{~K}$ ) carried out in polycrystalline (ss-) $\beta$ - $\mathrm{LiGaO}_{2}$ which was prepared using 
a solid-state synthesis route. A comparison to a powdered single crystal of ( $c r$-) $\beta$ - $\mathrm{LiGaO}_{2}$ is also presented in the case of the SAE experiments.

\section{Experimental}

\subsection{Materials}

\subsubsection{Solid-state synthesis of $\beta$ - $\mathrm{LiGaO}_{2}$}

Microcrystalline $\beta$ - $\mathrm{LiGaO}_{2}$ powder was prepared by reacting an appropriate mixture of $\mathrm{Li}_{2} \mathrm{CO}_{3}$ (99.997\%, Aldrich) and $\mathrm{Ga}_{2} \mathrm{O}_{3}(99.99 \%$, Aldrich) in a closed corundum crucible. After homogenizing the powders and pressing them to pellets, the samples were slowly heated up to $673 \mathrm{~K}$ and annealed for $1 \mathrm{~h}$. This was followed by two additional heating steps at $873 \mathrm{~K}(10 \mathrm{~h})$ and $1103 \mathrm{~K}$ ( 4 h). Finally, the pellets were quenched to ambient temperature by removing the pellets out of the furnace. Phase purity and the presence of the desired polymorph were confirmed by X-ray powder diffraction (PANalytical XPert PRO MPD, $\mathrm{Cu}-\mathrm{K} \alpha$ radiation). The pellets were ground in an argon glove-box to produce the sample $\left(s s-\mathrm{LiGaO}_{2}\right)$ used for the NMR experiments. The polycrystalline $s s-\mathrm{LiGaO}_{2}$ sample has an average particle size of $5 \mu \mathrm{m}$.

\subsubsection{Growth of $\beta$-LiGaO ${ }_{2}$ bulk crystals}

$\beta$ - $\mathrm{LiGaO}_{2}$ bulk crystals were grown using the conventional Czochralski technique with RF-heating and automatic diameter control. The starting materials $\mathrm{Li}_{2} \mathrm{CO}_{3}$ and $\mathrm{Ga}_{2} \mathrm{O}_{3}$ were of $99.99 \%$ purity. Both powders were dried, mixed in the stoichiometric ratio, sintered, pressed following standard procedures, and finally molten in a $40 \mathrm{~mL}$ crucible. $\mathrm{LiGaO}_{2}$ melts congruently at about $1585^{\circ} \mathrm{C}$. This high melting temperature requires the use of an iridium crucible. To decrease the melt evaporation, the temperature gradients in the growth set-up were adjusted by an active after-heater also consisting of iridium. The crystals were pulled along the $a$-axis with a rate of $1 \mathrm{~mm} / \mathrm{h}$ and a rotation rate of $15 \mathrm{rpm}$, and the growth atmosphere consisted of flowing nitrogen. The achieved single crystals were of about $60 \mathrm{~mm}$ in length and $15 \mathrm{~mm}$ in diameter. These crystals were finely ground using mortar and pestle in an argon glove-box to get the polycrystalline sample $\left(c r-\mathrm{LiGaO}_{2}\right)$ for the NMR experiments. The polycrystalline $\mathrm{cr}$ - $\mathrm{LiGaO}_{2}$ sample has an average particle size of $100 \mu \mathrm{m}$. 


\subsection{Solid-state NMR}

The solid-state NMR experiments were carried out using a Bruker Avance III 600 spectrometer, at a magnetic field strength of $14 \mathrm{~T}$, corresponding to Larmor frequencies of $233.30 \mathrm{MHz}$ and $183.07 \mathrm{MHz}$ for ${ }^{7} \mathrm{Li}$ and ${ }^{71} \mathrm{Ga}$, respectively. A $5 \mathrm{~mm}$ single-resonance probe has been used for static NMR measurements and a $2.5 \mathrm{~mm}$ double-resonance probe for magic-angle spinning (MAS) experiments. For static ${ }^{7} \mathrm{Li}$ experiments a standard solid-echo sequence has been used. For high-resolution MAS spectra, the samples were spun at $20 \mathrm{kHz}$ frequency. A very small tip angle of $\pi / 16$ was used for ${ }^{7} \mathrm{Li}$ and ${ }^{71} \mathrm{Ga}$ NMR. For all samples, the $T_{1}$ time was determined using saturation recovery experiments and recycle delays of $5 \times T_{1}$ were used. For $c r-$ $\mathrm{LiGaO}_{2}$, the SAE experiments were done with a starting saturation pulse train. The recycle delay used for ${ }^{7} \mathrm{Li} \mathrm{NMR}$ experiments for $s s-\mathrm{LiGaO}_{2}$ was of the order of tens of seconds. The ${ }^{7} \mathrm{Li}$ and ${ }^{71} \mathrm{Ga}$ NMR spectra were referenced ( $\left.0 \mathrm{ppm}\right)$ against dilute solutions of $\mathrm{LiCl}$ and $\mathrm{Ga}\left(\mathrm{NO}_{3}\right)_{3}$, respectively. The ${ }^{7} \mathrm{Li} \mathrm{SAE}$ decays were observed under static conditions with the standard Jeener-Broekaert pulse sequence [23]. The preparation time $\left(\tau_{\mathrm{p}}\right)$ of $15 \mu$ s was kept constant and the mixing time $\left(t_{\text {mix }}\right)$ was varied from $100 \mu$ s to $100 \mathrm{~s}$. The ${ }^{1} \mathrm{H}$ dipolar decoupling experiments were carried out with the robust frequency-swept SPINAL sequence [24, 25] with a radio-frquency strength of $150 \mathrm{kHz}$. All static variable-temperature NMR experiments were done on samples sealed in glass tubes and under dry nitrogen flow conditions.

\section{Results and discussion}

\section{1 ${ }^{71} \mathrm{Ga}$ and ${ }^{7} \mathrm{Li}$ NMR spectroscopy}

$\beta$ - $\mathrm{LiGaO}_{2}$ crystallizes in a distorted wurtzite type orthorhombic structure with a space group $P n a 2_{1}$ at ambient conditions [26]. The structure consists of $\mathrm{LiO}_{4}$ and $\mathrm{GaO}_{4}$ tetrahedra. Since there is only one crystallographic Ga site, this is reflected in the Ga NMR spectrum. Gallium has two naturally abundant NMR active isotopes, ${ }^{69} \mathrm{Ga}(60.108 \%)$ and ${ }^{71} \mathrm{Ga}(39.892 \%)$. Both the nuclei possess a quadrupole moment, as their spin number $(I=3 / 2)$ exceeds $1 / 2$. The interaction of the nuclear quadrupole moment with the electric field gradient (EFG) in the electronic surroundings of the nucleus is called the quadrupolar interaction. The magnitude of the quadrupolar interaction is measured using the quadrupolar coupling constant $\left(Q_{\mathrm{CC}}\right)$. The quadrupolar coupling constant can be defined as

$$
Q_{\mathrm{CC}}=\frac{V_{\mathrm{zz}} e Q}{h}
$$


where $V_{z z}$ is the largest component of the EFG tensor $V, e Q$ is the quadrupole moment and $h$ is the Planck constant. The asymmetry of the tensor $V$ is represented by the parameter $\eta_{Q}$,

$$
\eta_{\mathrm{Q}}=\frac{V_{\mathrm{xx}}-V_{\mathrm{yy}}}{V_{\mathrm{zz}}}
$$

The dependence of $Q_{\mathrm{CC}}$ and $\eta_{\mathrm{Q}}$ on the symmetry of the electronic surroundings helps to determine atomic coordination behaviors in structural studies. The magnetogyric ratio of ${ }^{71} \mathrm{Ga}\left(8.18 \times 10^{7} \mathrm{rad} \mathrm{T}^{-1} \mathrm{~s}^{-1}\right)$ is bigger than that of ${ }^{69} \mathrm{Ga}\left(6.44 \times 10^{7}\right.$

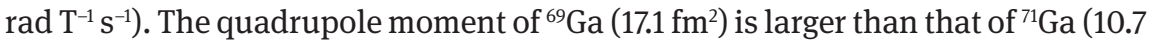
$\mathrm{fm}^{2}$ ), resulting in broader signals for ${ }^{69} \mathrm{Ga}$. Therefore, among these two nuclei, ${ }^{71} \mathrm{Ga}$ gives more resolved NMR signals, and can hence be used, for example, to confirm the absence of any significant impurity phase. ${ }^{71} \mathrm{Ga}$ NMR of both $\beta$ - $\mathrm{LiGaO}_{2}$ [27] and $\gamma$ - $\mathrm{LiGaO}_{2}$ (atmospheric pressure modification of $\alpha-\mathrm{LiGaO}_{2}$ ) [21] have been reported in literature. Figure $1 \mathrm{a}$ and $\mathrm{b}$ show the experimental central-transition ${ }^{71} \mathrm{Ga}$ spectra of $s s-\mathrm{LiGaO}_{2}$ and $c r-\mathrm{LiGaO}_{2}$, respectively. A single ${ }^{71} \mathrm{Ga}$ signal is observed for both the samples. The central-transition signals in both cases are broadened by the second-order quadrupolar interaction. Figure 1c shows a simulation of the

(a)

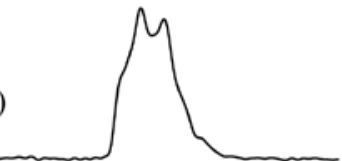

(b)

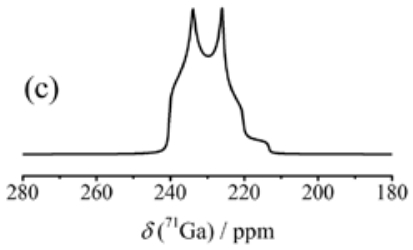

(d)

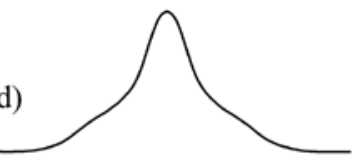

(e)

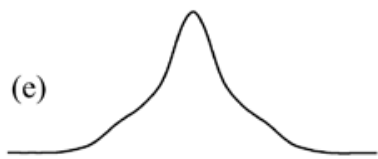

(f)

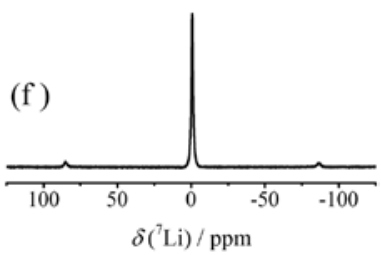

Fig. 1: On the left are the central transition ${ }^{71} \mathrm{Ga} N M R$ spectra of (a) $s s-\mathrm{LiGaO}_{2}$ and (b) $c r-\mathrm{LiGaO}_{2}$ under sample spinning (MAS) at a frequency of $20 \mathrm{kHz}$ and (c) simulated line-shape using $\delta_{\text {iso }}=241 \mathrm{ppm}, Q_{\mathrm{cC}}=3.8 \mathrm{MHz}$ and $\eta_{\mathrm{Q}}=0.4$. On the right are the static ${ }^{7} \mathrm{Li}$ NMR solid-echo spectra of (d) $s s-\mathrm{LiGaO}_{2},(\mathrm{e}) \mathrm{cr}$ - $\mathrm{LiGaO}_{2}$ and (f) the magic-angle spinning (at frequency of $20 \mathrm{kHz}$ ) spectrum of $s s-\mathrm{LiGaO}_{2}$. 
spectrum with the characteristic NMR parameters, such as $Q_{\mathrm{CC}}$ of $3.8 \mathrm{MHz}, \eta_{\mathrm{Q}}$ of 0.4 and isotropic chemical shift $\left(\delta_{\text {iso }}\right)$ of $241 \mathrm{ppm}$. These values are in very good agreement with the values for $\beta$ - $\mathrm{LiGaO}_{2}$ reported in literature [27]. The main difference between the ${ }^{71} \mathrm{Ga} N M R$ spectra of $s s-\mathrm{LiGaO}_{2}$ and $c r-\mathrm{LiGaO}_{2}$ is the rounding of the edges and steps of the second-order quadrupole pattern of the centraltransition signal in the case of $s s-\mathrm{LiGaO}_{2}$. This is mainly due to the large surface and increased number of defects in the sample prepared by solid-state synthesis, which induce distributions to NMR interactions, like chemical shielding, quadrupole coupling and/or dipolar coupling.

Solid-echo ${ }^{7} \mathrm{Li}$ NMR experiments carried out on $s s-\mathrm{LiGaO}_{2}$ and $c r-\mathrm{LiGaO}_{2}$ resulted in central (narrow contribution) as well as satellite (broad contribution) transition signals as shown in Figure 1d and e. The quadrupole coupling constant calculated from the largest splitting steps is $42 \mathrm{kHz}$ in both the cases, assuming $\eta_{\mathrm{Q}}=0$. The first-order quadrupole interactions get partially averaged with sample spinning at a frequency of $20 \mathrm{kHz}$, and the isotropic central transition and the remnant spinning-side bands can be seen for $s s-\mathrm{LiGaO}_{2}$ in Figure 1f. The ${ }^{7} \mathrm{Li}$ MAS NMR spectrum of $c r-\mathrm{LiGaO}_{2}$ (not shown) is similar to that of $s s-\mathrm{LiGaO}_{2}$.

\section{2 ${ }^{7}$ Li NMR motional narrowing}

The solid-echo ${ }^{7} \mathrm{Li} \mathrm{NMR}$ spectrum of $s s-\mathrm{LiGaO}_{2}$ shows a central transition signal, which is broadened mainly by Li-Li dipolar interactions. The FWHM of this signal changes slightly with temperature. Generally the FWHM has a high value at a lower temperature at the rigid lattice limit, and on complete averaging of dipolar interactions at high temperatures, it reaches the extreme narrowing limit. However, also in the present case, the reduction in FWHM with temperature can be the resultant of a dynamic process. The line-width changes from $6.3 \mathrm{kHz}$ to 5.4 $\mathrm{kHz}$ within the measurement temperature range (293-473 K). This is shown in Figure 2, together with a blown-up image (inset) with a solid-line to guide the eye. A complete motional averaging due to the full averaging of the dipolar interaction would decrease the FWHM drastically by at least one order of magnitude (like from a few $\mathrm{kHz}$ to a few hundreds of $\mathrm{Hz}$, as in the case of $\mathrm{LiAlO}_{2}$ [13]). But in the present study, the line-width reduction is only showing a change of a few hundreds of $\mathrm{kHz}$. This is ascribed to a partial averaging of dipolar interactions by the effect of a localized dynamic process, in which a subgroup of the Li ions are involved. A similar dynamic process has been observed in the past for $\mathrm{Li}_{15} \mathrm{Si}_{4}$ while probing ${ }^{7} \mathrm{Li}$ motional narrowing phenomena [28], and it has been attributed to limited Li diffusion due to geometrical restrictions. Localized motions usually represent toand-fro jump processes between an ionic site and a vacancy or an interstitial. 


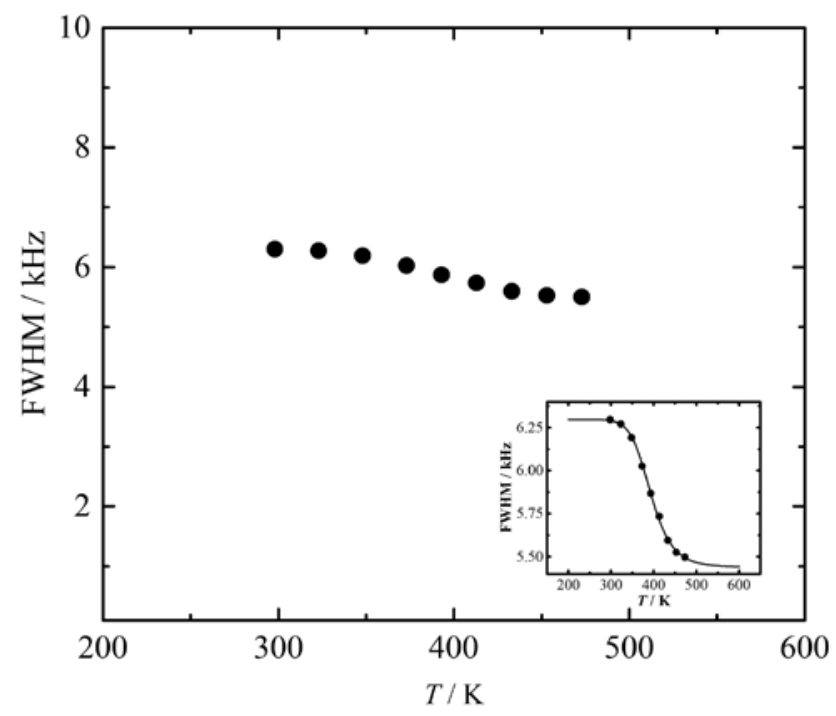

Fig. 2: The full-width at half-maximum of the central transition of static ${ }^{7}$ Li NMR spectra of ss-LiGaO ${ }_{2}$ plotted against the measurement temperature showing the motional narrowing corresponding to localized motion of Li ions. A blown-up image is shown on the inset with a solid-line to guide the eye.

But in the present case, the number of Li ions participating in the whole process is significantly small. Only approximately $1 \pm 0.5 \%$ of the total number of Li ions are involved in the localized dynamics, as estimated from the fraction of the NMR signal representing the mobile species. For these experiments, recording of fully relaxed ${ }^{~} \mathrm{Li}$ solid-echo spectra are necessary. For $\mathrm{ss}-\mathrm{LiGaO}_{2}$, exhibiting ${ }^{7} \mathrm{Li} T_{1}$ times of a few seconds, these variable-temperature experiments were possible, unlike in the case of $c r$ - $\mathrm{LiGaO}_{2}$ with $T_{1}$ times of the order of thousands of seconds. The large difference in $T_{1}$ times of the two samples is a resultant of two orders of difference in particle size, and the increased defect concentration in the sample prepared using solid-state synthesis. The absence of $\mathrm{H}$-related impurities in $\mathrm{LiGaO}_{2}$ was confirmed with ${ }^{1} \mathrm{H}$ NMR. In addition ${ }^{1} \mathrm{H}$-decoupling experiments were carried out during the ${ }^{7} \mathrm{Li}$ solid-echo experiments. No differences in ${ }^{7} \mathrm{Li}$ line-widths or intensities were observed in experiments with and without ${ }^{1} \mathrm{H}$ decoupling.

\section{3 ${ }^{7} \mathrm{Li}$ spin-alignment echo}

To study the localized motion on a different time scale, we have employed ${ }^{7} \mathrm{LiSAE}$ NMR experiments. Slow diffusion of ionic species can be investigated with SAE 
experiments, as shown in several previous reports [13, 29-31]. The advantage of the method is in the fact that direct estimation of ionic jump rates are possible from single-temperature measurements, which is not the case in relaxation measurements. This is carried out with the help of a two-dimensional array of SAE experiments with mixing-time dependence. The resultant echo amplitudes are plotted as a function of the mixing time $\left(t_{\text {mix }}\right)$, as shown in Figure 3, for ss $-\mathrm{LiGaO}_{2}$ at $373-448 \mathrm{~K}$. The jump rates $\left(\tau_{\mathrm{SAE}}^{-1}\right)$ are extracted from the curves using stretched exponential functions. The stretching exponents $(\gamma)$ were $0.49,0.50,0.50$ and 0.49 at $373,398,423$ and $448 \mathrm{~K}$, respectively. It is seen from the estimated jump rates that their magnitudes did not change significantly over the applied temperature range. But the same temperature range was influential in the ionic motion observed in the motional narrowing experiments. Therefore, the small changes in ionic jump rates are realistic in these conditions and they belong to the localized ion dynamics process.

Figure 4 shows the temperature dependence of the ionic jump rates in an Arrhenius plot and a linear fit is applied. The slope of the linear fit provides the Arrhenius activation energy $\left(E_{\mathrm{A}}\right)$ from the SAE experiments. In the case of ss$\mathrm{LiGaO}_{2}$, the estimated $E_{\mathrm{A}}$ is $0.13 \mathrm{eV}$, which is smaller compared to activation barriers in other Li-containing solids. This small magnitude of $E_{\mathrm{A}}$ is also indicating the presence of a localized motion in the $\mathrm{LiGaO}_{2}$ system. The extraction of diffusion coefficients are not desirable for such short to-and-fro jump processes, as they do not contribute to large scale translational diffusion. Since the ${ }^{7} \mathrm{Li} T_{1}$ time is relatively smaller for $s s$ - $\mathrm{LiGaO}_{2}$, the SAE experiments at higher temperatures were done in acceptable experiment times. For $\mathrm{Cr}-\mathrm{LiGaO}_{2}$, however, the SAE pulse program

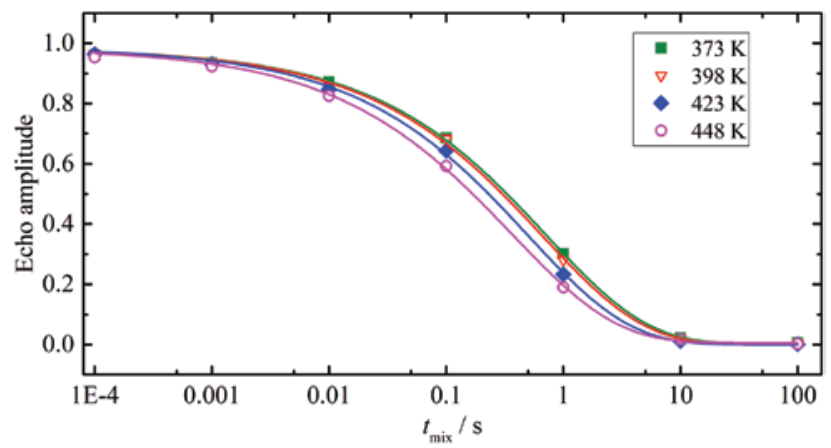

Fig. 3: Mixing-time $\left(t_{\text {mix }}\right)$ dependence of the ${ }^{7}$ Li spin-alignment echo NMR amplitudes of ss$\mathrm{LiGaO}_{2}$ at temperatures from $373 \mathrm{~K}$ to $448 \mathrm{~K}$. The stretched exponential fits yielded the correlation times $\left(\tau_{\mathrm{SAE}}\right)$ for different temperatures, where the stretching coefficients $(\gamma)$ remained close to 0.5 . 


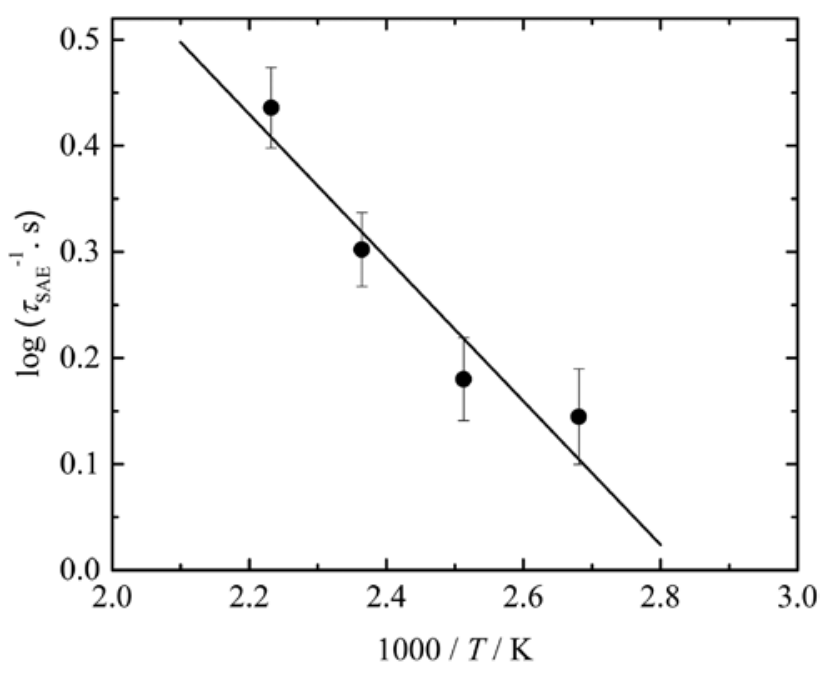

Fig. 4: The Arrhenius plot showing the dependence of ${ }^{7} \mathrm{Li} \tau_{\mathrm{SAE}}^{-1}$ of $\mathrm{Li}$ ions in $s s-\mathrm{LiGaO}_{2}$ with inverse temperature. The linear fit yielded an activation energy of $0.13 \mathrm{eV}$, corresponding to a localized Li motion in $\mathrm{LiGaO}_{2}$.

had to be modified to include saturation pulse train to shorten the duration of the experiments. The temperature dependent SAE jump rates in this case were fitted with a linear function which showed a higher Arrhenius activation energy $(0.40 \mathrm{eV})$. However, the $E_{\mathrm{A}}$ for overall ionic diffusion in $\mathrm{LiGaO}_{2}$ is expected to have similar magnitudes as that for $\mathrm{LiAlO}_{2}$ (i.e. $0.7 \mathrm{eV}$ ). This might also indicate the presence of a localized motion in $\mathrm{LiGaO}_{2}$ involving only a small number of $\mathrm{Li}$ ions.

\section{Conclusions}

Solid-state NMR spectroscopy methods have been employed to elucidate the localized $\mathrm{Li}$ ionic motion in polycrystalline $\mathrm{LiGaO}_{2}$. ${ }^{7} \mathrm{Li}$ motional narrowing of the central transition signal indicated a partially averaged Li-Li dipolar interaction pointing to local ionic motion in the temperature range between $300 \mathrm{~K}$ and $450 \mathrm{~K}$. In addition, ${ }^{7} \mathrm{Li}$ SAE experiments showed ionic jump rates between $10^{\circ}$ and $10^{1} \mathrm{~s}^{-1}$ (at $373-448 \mathrm{~K}$ ) with a very small activation energy $(0.13 \mathrm{eV})$ for $\mathrm{LiGaO}_{2}$, indicating a to-and-fro jump motion process.

Acknowledgements: The authors would like to express their gratitude for the financial support by Graduiertenkolleg Energiespeicher und Elektromobilität 
Niedersachsen (GEENI) and DFG Research Unit 1277 Mobilität von Lithium-Ionen in Festkörpern (molife).

\section{References}

1. M. S. Whittingham, Chem. Rev. 104 (2004) 4271.

2. J. B. Goodenough, Y. Kim, Chem. Mater. 22 (2010) 587.

3. N. Nitta, F. Wu, J. T. Lee, G. Yushin, Mater. Today 18 (2015) 252.

4. N. Roux, S. Tanaka, C. Johnson, R. Verall, Fusion Engin. Design 41 (1998) 31.

5. J. G. van der Laan, H. Kawamura, N. Roux, D. Yamaki, J. Nucl. Mater. 283-287 (2000) 99.

6. P. Waltereit, O. Brandt, K. H. Ploog, Appl. Phys. Lett. 75 (1999) 2029.

7. A. Kuhn, P. Sreeraj, R. Pöttgen, H.-D. Wiemhöfer, M. Wilkening, P. Heitjans, J. Am. Chem. Soc. 133 (2011) 11018.

8. D. Blanchard, A. Nale, D. Sveinbjörnsson, T. M. Eggenhuisen, M. H. W. Verkuijlen, Suwarno, T. Vegge, A. P. M. Kentgens, P. E. de Jongh, Adv. Funct. Mater. 25 (2015) 184.

9. C. V. Chandran, P. Heitjans, Annu. Rep. Nucl. Magn. Reson. Spectrosc. 89 (2016) 1.

10. C. V. Chandran, S. Pristat, E. Witt, F. Tietz, P. Heitjans, J. Phys. Chem. C 120 (2016) 8436.

11. K. Bösebeck, C. V. Chandran, B. K. Licht, M. Binnewies, P. Heitjans, Energy Technol. 12 (2016) 1598.

12. B. Ruprecht, M. Wilkening, R. Uecker, P. Heitjans, Phys. Chem. Chem. Phys. 14 (2012) 11974.

13. E. Witt, S. Nakhal, C. V. Chandran, M. Lerch, P. Heitjans, Z. Phys. Chem. 229 (2015) 1327.

14. D. Wiedemann, S. Nakhal, J. Rahn, E. Witt, M. M. Islam, S. Zander, P. Heitjans, H. Schmidt, T. Bredow, M. Wilkening, M. Lerch, Chem. Mater. 28 (2016) 915.

15. P. Heitjans, A. Schirmer, S. Indris, In: Diffusion in Condensed Matter - Methods, Materials, Models, P. Heitjans, J. Kärger (Eds.), Springer, Berlin (2005).

16. R. Boehmer, K. R. Jeffrey, M. Vogel, Prog. Nucl. Magn. Reson. 50 (2007) 87.

17. K. Funke, C. Cramer, D. Wilmer, In: Diffusion in Condensed Matter - Methods, Materials, Models, P. Heitjans, J. Kärger (Eds.), Springer, Berlin (2005).

18. D. Wohlmuth, V. Epp, P. Bottke, B. Bitschnau, I. Letofsky-Papst, M. Kriechbaum, H. Amenitsch, F. Hofer, M. Wilkening, I. Hanzu, J. Mater. Chem. A 2 (2014) 20295.

19. M. Marezio, J. P. Remeika, J. Phys. Chem. Solids 26 (1965) 1277.

20. R. Stoyanova, E. Zhecheva, G. Bromiley, T. B. Ballaran, R. Alcántara, J.-I. Corredor, J.-L. Tirado, J. Mater. Chem. 12 (2002) 2501.

21. E. Zhecheva, R. Stoyanova, R. Alcántara, J. L. Tirado, J. Phys. Chem. B 107 (2003) 4290.

22. R. Stoyanova, E. Zhecheva, R. Alcántara, J.-L. Tirado, G. Bromiley, F. Bromiley, T. B. Ballaran, J. Mater. Chem. 14 (2004) 366.

23. J. Jeener, P. Broekaert, Phys. Rev. 157 (1967) 232.

24. C. V. Chandran, T. Bräuniger, J. Magn. Reson. 200 (2009) 226.

25. C. V. Chandran, G. Hempel, T. Bräuniger, Solid State Nucl. Magn. Reson. 40 (2011) 84.

26. G. M. Kuz'micheva, V. B. Rybakov, A. V. Gaister, E. V. Zharikov, Inorg. Mater. (USSR) 37 (2001) 281.

27. J. T. Ash, P. J. Grandinetti, Magn. Reson. Chem. 44 (2006) 823. 
28. S. Dupke, T. Langer, R. Pöttgen, M. Winter, S. Passerini, H. Eckert, Phys. Chem. Chem. Phys. 14 (2012) 6496.

29. M. Wilkening, W. Küchler, P. Heitjans, Phys. Rev. Lett. 97 (2006) 065901.

30. M. Wilkening, P. Heitjans, J. Phys. Condens. Mater. 18 (2006) 9849.

31. M. Wilkening, A. Kuhn, P. Heitjans, Phys. Rev. B 78 (2008) 054303. 